\title{
Sensitivity and rapidity of blood culture bottles in the detection of cornea organ culture media contamination by bacteria and fungi
}

\author{
G Thuret, A Carricajo, C Chiquet, A C Vautrin, N Celle, M Boureille, S Acquart, \\ G Aubert, J Maugery, P Gain
}

See end of article for authors' affiliations

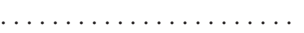

Correspondence to: Professor Philippe Gain, Service d'Ophtalmologie (pavillon 50A), CHRU de Bellevue, 25 Boulevard Pasteur, F 42055

Saint-Etienne Cedex 2 ,

France; philippe.gain@ univ-st-etienne.fr

Accepted for publication 16 July 2002

\begin{abstract}
Aims: To test the bactericidal activity of standard organ culture medium, and to compare the sensitivity and rapidity of blood culture bottles with conventional microbiological methods for detection of bacteria and fungi inoculated in a standard cornea organ culture medium.

Methods: The bactericidal activity of contaminated standard organ culture medium containing 100 $\mathrm{IU} / \mathrm{ml}$ penicillin, $0.1 \mathrm{mg} / \mathrm{ml}$ streptomycin, and $0.25 \mu \mathrm{g} / \mathrm{ml}$ amphotericin B was evaluated after 48 hours of incubation at $31^{\circ} \mathrm{C}$ with five inocula of 14 bacteria. Two yeasts (Candida spp) and one Aspergillus were also tested. Contaminated media were then inoculated in three blood bottles (aerobic, anaerobic, fungal) placed in a Bactec 9240 automat; three conventional microbiological broths were the control. Changes in colour of organ culture medium and growth on conventional broth were screened daily by visual inspection. The sensitivity and rapidity of detection of contamination were compared between the three methods: blood bottle, conventional, and visual.

Results: Organ culture medium eradicated five bacteria irrespective of the starting inoculums: Streptococcus pneumoniae, Branhamella catarrhalis, Escherichia coli, Propionibacterium acnes, and Haemophilus influenzae. For micro-organisms where the medium was ineffective or bactericidal only (methicillin resistant Staphylococcus aureus, methicillin sensitive Staphylococcus aureus, Staphylococcus epidermidis, Staphylococcus haemolyticus, Pseudomonas aeruginosa, Acinetobacter baumannii, Bacillus subtilis, Klebsiella pneumoniae, Enterococcus faecalis, Candida albicans, Candida kruzei, Aspergillus fumigatus), the blood bottle, conventional, and visual methods detected microbial growth in $100 \%, 76.5 \%$, and $70 \%$ of cases respectively. Mean detection time using blood bottles was 15.1 hours (SD 13.8, range 2-52). In cases of detection by the blood bottle method and the conventional method, the former was always faster: $95.5 \%$ against $65.2 \%$ detection within 24 hours ( $p=0.022$ ) respectively.

Conclusions: Blood bottles detect more efficiently and more rapidly a wider range of bacteria and fungi than the conventional microbiological method and the visual inspection of organ culture media.
\end{abstract}

O rgan culture is the most common cornea storage technique in Europe. ${ }^{1}$ Its advantages include the possibility of doing one or more microbiological tests that minimise the risk of transmitting a bacterial or fungal infection to the recipient. ${ }^{2}$ Given the severity of cases of early endophthalmitis after penetrating keratoplasty (PKP), every effort must be made to limit the risk of micro-organism proliferation during storage, and to optimise the microbiological safety tests.

Given that most micro-organisms grow well at high temperature $\left(31^{\circ} \mathrm{C}-37^{\circ} \mathrm{C}\right.$, depending on the cornea bank $\left.{ }^{1}\right)$, and that antibiotics are active at these temperatures, most organ culture media used in Europe, whether made commercially or by the cornea banks, contain two antibiotics (typically penicillin and streptomycin) and an antifungal (usually amphotericin B). These anti-infectious agents partially eradicate, or at least limit the growth of, micro-organism contaminants. In addition, organ culture media contain phenol red, an indicator whose colour varies with $\mathrm{pH}$, most often denoting contamination. ${ }^{3}$

Most cornea banks use a conventional microbiological method to detect the presence of aerobic bacteria, anaerobic bacteria, or fungi. When this is combined with daily visual inspection of medium colour, cornea can be delivered with satisfactory microbiological safety, after a microbiological "quarantine" of 10-12 days depending on the bank'; there is no consensus. This period is needed to detect, using a conven- tional microbiological method, residual contamination not eradicated by the antiobiotics in the organ culture medium.

Blood bottles detect contaminations more sensitively and more rapidly than conventional microbiological methods, ${ }^{45}$ particularly for liquids with low inoculum levels and/or that already contain antibiotics. This has been demonstrated for liquid from ascites, ${ }^{6}$ peritoneal dialysis, ${ }^{78}$ articular, ${ }^{90}$ and cerebrospinal fluid. ${ }^{11}$ We thus thought it logical to use them to test the sterility of cornea organ culture media. In our previous pilot study (although this was not comparative) they seemed to detect, reliably and rapidly, contamination of organ culture media. ${ }^{12}$

The purpose of this study was therefore to compare this protocol with the conventional microbiological method routinely used by most of the cornea banks. Because contamination in our cornea bank is rare, the study was done in vitro by seeding organ culture media with a wide range of micro-organisms responsible for postoperative endophthalmitis. We conducted two series of experiments. The first series tested the bactericidal effect of organ culture medium on bacterial and fungal inocula. The second compared how sensitively and quickly residual contamination was detected by the three methods: daily visual inspection of the medium colour ("visual method"), the conventional microbiological method ("conventional method"), and blood culture bottles ("blood bottle method"). 


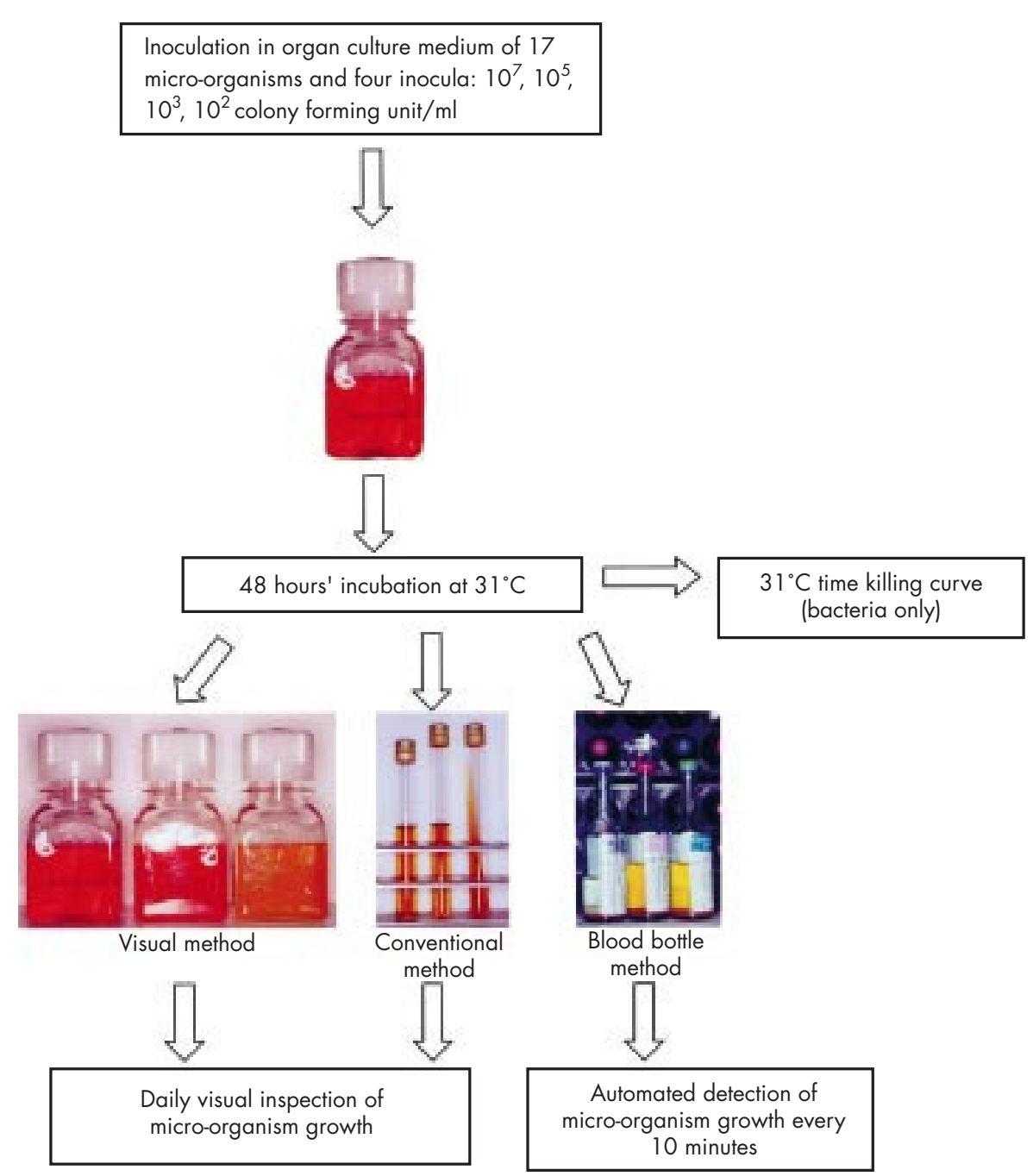

Figure 1 Design of our study.

micro-organisms and four inocula: $10^{7}, 10^{5}$

$10^{3}, 10^{2}$ colony forming unit/m

Figur

\section{MATERIALS AND METHODS}

The design of our experiments is presented in Figure 1.

\section{Micro-organisms}

Bacterial and fungal strains were obtained from the American Type Culture Collection (ATCC) (Rockville, MD, USA) or from corneal swabs and scrapings submitted for culture to the microbiology department of our hospital. Fourteen bacteria were tested: methicillin sensitive Staphylococcus aureus ATCC 25923, one methicillin resistant Staphylococcus aureus, Staphylococcus epidermidis ATCC 12228, one Staphylococcus haemolyticus, Streptococcus pneumoniae ATCC 49619, Enterococcus faecalis ATCC 29212, Haemophilus influenzae ATCC 49247, Branhamella catarrhalis ATCC 2631, Escherichia coli ATCC 25922, Klebsiella pneumoniae ATCC 104, Pseudomonas aeruginosa ATCC 27853, Acinetobacter baumannii ATCC 19606, Bacillus subtilis ATCC 6633, and one Propionibacterium acnes. Two yeasts and one filamentous fungus were tested: Candida albicans ATCC 90028, Candida krusei ATCC 6258, and Aspergillus fumigatus ATCC 13935.

\section{Bactericidal activity of the organ culture medium}

The bactericidal activity of a commercial medium (Inosol, Chauvin-Opsia, Toulouse, France) containing $100 \mathrm{IU} / \mathrm{ml}$ penicillin $\mathrm{G}, 0.1 \mathrm{mg} / \mathrm{ml}$ streptomycin and $0.25 \mu \mathrm{g} / \mathrm{ml}$ amphotericin B, was measured on four inocula of the 17 micro-organisms. An overnight culture in Mueller-Hinton broth was briefly diluted in the organ culture medium to give
$10^{7}, 10^{5}, 10^{3}$, and $10^{2}$ colony forming unit $/ \mathrm{ml}(\mathrm{cfu} / \mathrm{ml})$. A $100 \mu \mathrm{l}$ aliquot was removed, serial diluted, and plated on MuellerHinton agar to determine the exact starting concentration of bacteria and fungi. The same amount of inoculum was added to Mueller-Hinton broth as a growth control. For the 14 bacteria, $0.1 \mathrm{ml}$ of the organ culture medium incubated at $31^{\circ} \mathrm{C}$ was then removed at fixed times $(2,6,24$, and 48 hours $)$ and plated on Mueller-Hinton agar (except for Streptococcus pneumoniae, Branhamella catarrhalis, and Propionibacterium acnes, where chocolate media were used). The number of colonies was counted after 48 hours' incubation at $35^{\circ} \mathrm{C}$ and the results plotted on semilogarithmic graph paper. A bactericidal effect, defined as a $4 \log { }_{10}$ decrease in $\mathrm{cfu} / \mathrm{ml}$, corresponding to survival of less than $0.01 \%$ of the micro-organisms, was measured for the $10^{7}$ and $10^{5}$ inocula. Eradication was defined as a lack of surviving micro-organisms in the $10^{3}$ and $10^{2}$ $\mathrm{cfu} / \mathrm{ml}$ inocula. The $10^{2} \mathrm{cfu} / \mathrm{ml}$ inoculum was chosen to assess how well the three methods detect very low contamination. Each inoculum for each micro-organism was tested three times. Given the very low concentration of amphotericin B in the organ culture medium, fungicidal activity was not tested.

The inoculated organ culture media were incubated in sealed flasks for 48 hours at $31^{\circ} \mathrm{C}$ in a conventional carbon dioxide free dry incubator, identical to that used in our cornea bank. In most European banks ${ }^{1}$ corneas routinely spend this period in the so called "transport" medium before the first endothelial and microbiological tests are done and the medium changed. 
Table 1 Bactericidal activity and eradication capacity of organ culture medium according to the bacteria and the inoculum. Because of the definition of bactericidal activity, ${ }^{*}$ for $10^{3}$ and $10^{2}$ inocula, only eradication was checked

\begin{tabular}{|c|c|c|c|c|c|c|}
\hline \multirow[b]{3}{*}{ Bacteria } & \multicolumn{6}{|l|}{ Inoculum } \\
\hline & \multicolumn{2}{|l|}{$10^{7}$} & \multicolumn{2}{|l|}{$10^{5}$} & \multirow{2}{*}{$\begin{array}{l}10^{3} \\
\text { Eradication }\end{array}$} & \multirow{2}{*}{$\begin{array}{l}10^{2} \\
\text { Eradication }\end{array}$} \\
\hline & Bactericidal* & Eradication $\dagger$ & Bactericidal & Eradication & & \\
\hline Methicillin resistant Staphylococcus aureus & no & no & no & no & no & no \\
\hline Methicillin sensitive Staphylococcus aureus & yes & no & yes & no & yes & yes \\
\hline Staphylococcus epidermidis & no & no & no & no & yes & yes \\
\hline Staphylococcus haemolyticus & yes & no & yes & yes & yes & yes \\
\hline Pseudomonas aeruginosa & no & no & no & no & yes & yes \\
\hline Acinetobacter baumannii & no & no & no & no & yes & yes \\
\hline Bacillus subtilis & no & no & yes & yes & yes & yes \\
\hline Klebsiella pneumoniae & no & no & yes & no & yes & yes \\
\hline Enterococcus faecalis & yes & no & yes & no & yes & yes \\
\hline Streptococcus pneumoniae & yes & yes & yes & yes & yes & yes \\
\hline Branhamella catarrhalis & yes & yes & yes & yes & yes & yes \\
\hline Escherichia coli & yes & yes & yes & yes & yes & yes \\
\hline Propionibacterium acnes & yes & yes & yes & yes & yes & yes \\
\hline Haemophilus influenzae & yes & yes & yes & yes & yes & yes \\
\hline
\end{tabular}

\section{Sensitivity and rapidity of the three methods in detecting contamination}

In the visual method, changes in colour (to orange or yellow) or in turbidity of the organ culture medium, indicating positivity, were screened daily for 14 days by visual inspection.

In the conventional method, $1 \mathrm{ml}$ of contaminated organ culture medium was inoculated in a brain-heart broth $(10 \mathrm{ml})$ and a Schaedler broth $(10 \mathrm{ml})$ incubated at $35^{\circ} \mathrm{C}$ and in one Sabouraud agar $(10 \mathrm{ml})$ incubated at $28^{\circ} \mathrm{C}$. Growth on conventional broth was screened daily by visual inspection for 14 days.

In the blood bottle method, $2.5 \mathrm{ml}$ of contaminated organ culture medium was injected into one Bactec Plus Aerobic/F bottle $(25 \mathrm{ml})$, one Bactec Lytic/10 Anaerobic/F bottle $(40 \mathrm{ml})$, and one Bactec Mycosis IC/F (40 ml) (Becton Dickinson, Cockeysville, MD, USA). The bottles were placed in a Bactec 9240 incubator for 14 days at $35^{\circ} \mathrm{C}$ and rocked continuously. As described in a previous paper, ${ }^{12}$ the incubator detected any rise in carbon dioxide produced by bacterial growth. A sensor placed at the bottom of each bottle reacted with the carbon dioxide and produced fluorescence proportional to the carbon dioxide level. Fluorescence was measured every 10 minutes and time to detection was rounded to the nearest hour.

In all three methods, identification of isolate microorganism was verified from subcultures of the positive media by standard bacteriological methods. The inoculated microorganism always corresponded to the one detected, thus ruling out any exogenous contamination during manipulation by the technician.

\section{RESULTS}

\section{Bactericidal activity of the organ culture medium (Table} 1)

The antibiotics in the organ culture medium eradicated five bacteria irrespective of the starting inoculum: Streptococcus pneumoniae, Branhamella catarrhalis, Escherichia coli, Propionibacterium acnes, and Haemophilus influenzae. The antibiotics of the organ culture medium had no effect on the methicillin resistant Staphylococcus aureus. Only the $10^{2}, 10^{3}$, and $10^{5}$ inocula of Staphylococcus haemolyticus and Bacillus subtilis were eradicated.

\section{Sensitivity and rapidity of the three methods in} detecting residual contamination (Table 2)

Detection of residual contamination was $100 \%$ by the blood bottles $(30 / 30), 76.5 \%$ by the conventional method (23/30), and only $70 \%$ by the visual method $(21 / 30)$. No blood bottle tested positive without an identifiable germ, thus ruling out the possibility of a false positive having been generated by a physicochemical reaction of the organ culture medium with the contents of the bottle. When both the conventional and the blood bottle methods detected contamination $(n=23)$, the latter was significantly quicker: positivity was nearly always observed within 24 hours (95.5\%, 22/23 against 65.2\%, 15/23) (Fisher exact test, $\mathrm{p}=0.022$ ). Blood bottles also detected contamination within 8 hours in $40 \%$ of cases (12/30), corresponding to the technicians' shift length. Mean time to contamination detection by the blood bottles was 15.1 hours (SD 13.8, range 2-52): 19.5 hours for the bacteria (SD 14.2, range 3-52), and only 3 hours (SD 0.4, range 2-4) for the yeasts.

\section{DISCUSSION}

Early post-PKP endophthalmitis may be linked to the recurrence of an infection present in the recipient before grafting, to peroperative contamination, or to transmission of a micro-organism via contaminated graft tissue. Organ culture at $31^{\circ} \mathrm{C}, 34^{\circ} \mathrm{C}$, or $37^{\circ} \mathrm{C}$, because of the presence of antibiotics active at these temperatures, coloured $\mathrm{pH}$ indicators and microbiological tests, eradicates micro-organisms or at least detects residual survival. It thus made sense, in the first series of experiments, to test the bactericidal strength of the organ culture medium. We used an original microbiological protocol that adapts techniques for studying the kinetics of antibiotic activity (time killing curves) to the real cornea storage temperature $\left(31^{\circ} \mathrm{C}\right.$ in our bank). The second series of experiments compared how effectively and quickly residual contamination is detected by three methods: a bacteriological method thought more suitable for low inocula and/or when antiobiotics are present, ${ }^{12}$ a conventional microbiological method, and daily visual inspection of the colour of organ culture media.

The micro-organisms studied were chosen because they are implicated in early post-PKP endophthalmitis ${ }^{13}$ or because they are typically present in postmortem eye flora. ${ }^{14}{ }^{15}$ The combinations of antibiotics and antifungals used in organ culture media in Europe are similar. The 2002 survey of 78 cornea banks by the European Eye Bank Association ${ }^{1}$ showed that 68\% (53/78) used organ culture alone or combined with storage at $4^{\circ} \mathrm{C}$. Penicillin was combined with streptomycin in $87 \%$ of cases $(46 / 53)$, nearly always in concentrations of $100 \mathrm{IU} / \mathrm{ml}$ and $0.1 \mathrm{mg} / \mathrm{ml}$ respectively. According to the same survey, an antifungal is always used: amphotericin B is most common (92.5\%, 49/53 
Table 2 Mean time to detection (hours) of residual contamination of organ culture medium by the three methods: visual, conventional and blood bottles. Of 14 bacteria initially inoculated, five were fully eradicated by the antibiotics in the organ culture medium and therefore did not appear in this table. Of the remaining nine bacteria and three fungi, only the inocula presenting residual growth after the 48 hour incubation in the organ culture medium are presented. Detection times corresponded to the interval between inoculation and detection of a change in turbidity and/or colour using the visual method and the conventional microbiological method, and to automat detection of growth using the blood bottle method. Identification of micro-organisms required each time a further 24-48 hours. All three methods detected contamination of the fungi, but the quickest results were obtained by the fungal or aerobic blood bottles

\begin{tabular}{|c|c|c|c|}
\hline \multirow[b]{2}{*}{$\begin{array}{l}\text { Micro-organism } \\
\text { Inoculum }\end{array}$} & \multicolumn{3}{|c|}{ Time between inoculation and positivity (hours) } \\
\hline & $\begin{array}{l}\text { Visual method (aspect } \\
\text { of the medium) }\end{array}$ & $\begin{array}{l}\text { Conventional method } \\
\text { (mean, triplicate) }\end{array}$ & $\begin{array}{l}\text { Blood bottle method (mean, } \\
\text { triplicate) }\end{array}$ \\
\hline \multicolumn{4}{|c|}{ Methicillin resistant Staphylococcus aureus } \\
\hline $10^{7}$ & 48 (turbid) & $48(48-48-48)$ & $17.7(19-17-17)$ \\
\hline $10^{5}$ & no & $80(72-48-120)$ & $19.3(13-22-23)$ \\
\hline $10^{3}$ & no & no & $36(28-48-32)$ \\
\hline $10^{2}$ & no & no & $39.3(38-37-43)$ \\
\hline \multicolumn{4}{|c|}{ Methicillin sensitive Staphylococcus aureus } \\
\hline $10^{7}$ & no & no & $19.7(16-25-18)$ \\
\hline $10^{5}$ & no & no & $37.7(33-52-28)$ \\
\hline \multicolumn{4}{|c|}{ Staphylococcus epidermidis } \\
\hline $10^{7}$ & 24 (yellow) & $24(24-24-24)$ & $3(3-3-3)$ \\
\hline $10^{5}$ & 72 (yellow) & $24(24-24-24)$ & $16(21-4-23)$ \\
\hline \multicolumn{4}{|c|}{ Staphylococcus haemolyticus } \\
\hline $10^{7}$ & no & no & $43.3(38-40-52)$ \\
\hline \multicolumn{4}{|c|}{ Pseudomonas aeruginosa } \\
\hline $10^{7}$ & 24 (turbid) & $24(24-24-24)$ & $3.3(3-3-4)$ \\
\hline $10^{5}$ & 24 (turbid) & $24(24-24-24)$ & $8.6(7-4-15)$ \\
\hline \multicolumn{4}{|c|}{ Acinetobacter baumannii } \\
\hline $10^{7}$ & 24 (turbid) & $32(48-24-24)$ & $5(3-8-4)$ \\
\hline $10^{5}$ & 24 (turbid) & $32(48-24-24)$ & $4(3-4-5)$ \\
\hline \multicolumn{4}{|l|}{ Bacillus subtilis } \\
\hline $10^{7}$ & 96 (yellow) & $48(48-48-48)$ & $13(12-14-13)$ \\
\hline \multicolumn{4}{|c|}{ Klebsiella pneumoniae } \\
\hline $10^{7}$ & no & $48(24-72-48)$ & $14.7(20-13-11)$ \\
\hline $10^{5}$ & no & no & $20.7(24-18-20)$ \\
\hline \multicolumn{4}{|c|}{ Enterococcus faecalis } \\
\hline $10^{7}$ & 48 (yellow) & $48(48-48-48)$ & $14.7(25-15-4)$ \\
\hline $10^{5}$ & no & no & $35(30-25-50)$ \\
\hline \multicolumn{4}{|l|}{ Candida albicans } \\
\hline $10^{7}$ & 24 (orange) & $24(24-24-24)$ & $3(3-3-3)$ \\
\hline $10^{5}$ & 24 (orange) & $24(24-24-24)$ & $3(3-3-3)$ \\
\hline $10^{3}$ & 24 (orange) & $24(24-24-24)$ & $3(3-3-3)$ \\
\hline $10^{2}$ & 24 (orange) & $24(24-24-24)$ & $3(4-2-3)$ \\
\hline \multicolumn{4}{|l|}{ Candida krusei } \\
\hline $10^{7}$ & 24 (yellow) & $24(24-24-24)$ & $2.7(2-3-3)$ \\
\hline $10^{5}$ & 24 (yellow) & $24(24-24-24)$ & $3(3-3-3)$ \\
\hline $10^{3}$ & 24 (yellow) & $24(24-24-24)$ & $3(3-3-3)$ \\
\hline $10^{2}$ & 24 (orange) & 24 (24-24-24) & 3. $(4-3-3)$ \\
\hline \multicolumn{4}{|c|}{ Aspergillus fumigatus } \\
\hline $10^{7}$ & 48 (turbid) & $24(24-24-24)$ & $11.3(13-15-6)$ \\
\hline $10^{5}$ & 48 (turbid) & $24(24-24-24)$ & $15.7(17-15-15)$ \\
\hline $10^{3}$ & 48 (turbid) & $24(24-24-24)$ & $20(20-17-23)$ \\
\hline $10^{2}$ & 48 (turbid) & $40(24-72-24)$ & $27.7(17-50-16)$ \\
\hline
\end{tabular}

cases) although concentrations differ between a trace $(0.25$ $\mu \mathrm{g} / \mathrm{ml})$ in $77.5 \%$ of cases $(38 / 49)$, low $(2.5 \mu \mathrm{g} / \mathrm{ml})$ in $18.5 \%$ of cases $(9 / 49)$, and high $(25 \mu \mathrm{g} / \mathrm{ml})$ in two banks $(4 \%)$. The combination of penicillin and streptomycin was thus chosen in our experiment because they are very common. This combination was highly effective, eradicating five bacteria. This result agrees with previous studies of similar organ culture media. ${ }^{16}$ Only Borderie, studying the same medium as ours, found an Escherichia coli multiresistant to antibiotics. ${ }^{17}$ No case of early post-PKP endophthalmitis caused by this micro-organism has been reported in the literature. This bacterium was thus not tested in our study which makes comparison difficult. Of the bacteria eradicated by the antibiotics, cases of post-PKP endophthalmitis are conventionally attributed to Streptococcus pneumoniae: $8 / 59$ cases in Kloess's review. ${ }^{13}$ In that review, however, its transmission via the grafted cornea was proved in only two cases; more importantly, the corneas were stored at $4^{\circ} \mathrm{C}$ in a medium not containing a penicillin active on this organism. ${ }^{18}$
Similarly, Propionibacterium acnes and Streptococcus pneumoniae have been found in corneoscleral rims, but only after storage at $4^{\circ} \mathrm{C}^{13} 1920$

Our experimental study deliberately used no corneas. Some Staphylococcus spp and Streptococcus spp can adhere to tissues and secrete a mucoid substance called "slime" that protects them from antibiotics. ${ }^{21}{ }^{22}$ This may have influenced the results of the first part of the study, on the bactericidal activity of the media. However, inoculation of the micro-organisms would not have been "natural": both endothelial and epithelial surfaces would have been exposed, whereas true contaminations typically come from the ocular surface. Moreover, experimental contamination of the corneas would not have allowed accurate testing of the inoculum actually added to the organ culture medium. Lastly, Albon and Wiffen have shown there is no link between contamination of the corneoscleral rim and transmission of infection to the recipient. ${ }^{1923}$ 
The superiority of blood bottles appears clearly. We showed them to be more sensitive than the conventional microbiological method and the visual method. They detected a wider spectrum of bacteria, sometimes even with high inocula missed by the other two techniques. Methicillin sensitive Staphylococcus aureus at $10^{7}$ and $10^{5} \mathrm{cfu} / \mathrm{ml}$, Staphylococcus haemolyticus at $10^{7} \mathrm{cfu} / \mathrm{ml}$, Klebsiella pneumoniae at $10^{5} \mathrm{cfu} / \mathrm{ml}$, and Enterococcus faecalis at $10^{5} \mathrm{cfu} / \mathrm{ml}$ were never detected by the conventional method and induced no change of colour of the organ culture medium. Hughes demonstrated that culture using blood bottle methods detected significantly more pathogens than conventional methods, especially of Staphylococcus and Enterococcus spp. ${ }^{24}$ This is important in ophthalmology because these are among the main organisms responsible for post-PKP endophthalmitis. ${ }^{13}$

In cases of low inocula, the high sensitivity of blood bottles was predictable. The lack of sensitivity of the conventional microbiological method may be due to antibiotics contained in the organ culture media and which may inhibit the growth of the micro-organism in culture. Resins, such as those present in Bactec Plus Aerobic/F and Bactec Lytic/10 Anaerobic/F bottles, may reduce the inhibiting effects of antibiotics. ${ }^{24}$ It is likely that these low inocula would have been eradicated when the medium was changed: fresh antibiotics are then added, an important point because their action is limited in time. ${ }^{25}$ Lastly, it is likely that a very low inoculum has no contaminating potential in the recipient because of the spontaneous bactericidal activity of the aqueous humour ${ }^{26}$ and because of the perioperative antibiotics systematically prescribed. Highly sensitive detection methods, such as Albon's, subjecting corneoscleral rims to mechanical extraction in a Stomacher blender, ${ }^{23}$ detected micro-organisms in $28.9 \%$ of cases, whereas microbiological testing of organ culture media found them in only $0.53 \%{ }^{25}$ to $0.7 \%{ }^{17}$ of cases. Yet Albon, in her series, reported no endophthalmitis. In our study, low inocula of $10^{3}$ and $10^{2} \mathrm{cfu} / \mathrm{ml}$ of methicillin resistant Staphylococcus aureus were detected only by blood bottles, in 36 and 39.3 hours respectively, and would probably have been eradicated at the renewal of organ culture medium. We think that, in this respect, the lowering of the sensitivity threshold is more useful for quality control of the entire storage procedure, from procurement to graft, than to surgeons as a predictor of postoperative infection.

Given the very low concentration and the instability of amphotericin $\mathrm{B}^{27}$ which is used mainly to ensure fungicidal action during production of the organ culture medium, we felt it unnecessary to test the fungicidal activity of the organ culture medium. We were reinforced in this choice since the three fungi grew and were detected after the 48 hour incubation in the organ culture medium irrespective of the inoculum. The rarity yet severity of fungal endophthalmitis, ${ }_{1}^{1328}$ and the slowness of growth for some species, naturally led us to optimise our fungal tests. In our previous study, ${ }^{12}$ we showed that the Bactec Plus Aerobic/F bottle combined with the Bactec Lytic/10 Anaerobic/F bottle were able to detect Candida spp effectively and quickly (5/5 cases, all within 48 hours). The present study aimed to assess the utility of the Bactec Mycosis IC/F blood bottle, specially developed for fungal detection. Fricker-Hidalgo, in a study of 43 strains from 10 species, demonstrated that, though no more sensitive, the fungal bottle saved time compared to aerobic and anaerobic blood bottles. ${ }^{29}$ Moreover, a selective fungal medium is necessary when yeasts and bacteria are present, as bacteria may inhibit yeast growth. ${ }^{29}$ In our study, the small number of fungi tested allowed no conclusion as to the comparative utility of Mycosis IC/F bottles. In general terms, although the three methods (blood bottle, conventional, visual) detected fungal contamination, the blood bottles revealed contaminations by Candida spp much faster (typically within 3 hours) irrespective of the inoculum.

As for the speed of detection of the three methods, we are well aware that comparative studies of this type contain one bias that cannot be eliminated in a normal laboratory, where standardised protocols for cultures must be strictly followed: the reading frequency differed between the three methods. In the blood bottle method, reading was done automatically every 10 minutes. For the two other methods, reading was once daily to comply with cornea bank and bacteriology laboratory practice, whereby the technicians inspect the organ culture bottles and conventional microbiological media only once daily. This consolidates the utility of the blood bottles, which detected contamination within 8 hours in nearly half the cases, corresponding to the technicians' shift length. Early elimination from storage of contaminated corneas, particularly due to yeasts, minimises the risk of contamination of the other corneas in the incubator and limits storage costs. In addition, replacement of the conventional method with an automated culture system like the Bactec blood bottle method significantly reduced labour requirements in our bacteriology laboratory; and, despite the extra cost of the three blood bottles (approximately $€ 10 v € 1.9 ; £ 6.3 v £ 1.2$ ), yielded substantial savings. Lastly, our use of this protocol at the start and end of organ culture allows faster delivery of corneas without affecting their microbiological safety, and our patients benefit from corneas of higher endothelial cell density.

\section{CONCLUSION}

Use of this more sensitive protocol should, in theory, cause a higher cornea discard rate. But in practice it is likely that, because there are fewer operation steps performed in better conditions (blood bottles are sealed, and specimens can be directly injected under laminar flow hood in the cornea banks or operating theatre), this effect is offset by a fall in exogenous contamination. The contamination rate of $3.2 \%(30 / 934$ corneas), obtained by this protocol since its introduction in our cornea bank and by the favourable clinical outcome in our patients (one hypopyon out of 412 penetrating keratoplasty), is most acceptable.

We consider that the greater sensitivity and speed, at no extra cost, of blood bottles in testing the microbiological safety of organ culture media compared to conventional microbiological methods, justifies their general introduction for routine use.

\section{ACKNOWLEDGEMENTS}

We thank the "Direction de la Recherche Clinique de Saint-Etienne (Pr Hervé Decousus)" for funding (PHRC research programme).

Proprietary interest: the authors do not have any proprietary interest in the materials used in this study.

Part of this paper was presented at the 14th annual meeting of the European Eye Bank Association, held on 18-19 January 2002 in Paris, France.

Authors' affiliations

G Thuret, C Chiquet, J Maugery, P Gain, Department of

Ophthalmology, Bellevue Hospital, 25 Bd Pasteur, F 42055 Saint-Etienne Cedex 2, France

A Carricajo, A C Vautrin, N Celle, M Boureille, G Aubert, Department of Microbiology, Bellevue Hospital, 25 Bd Pasteur, F 42055 Saint-Etienne Cedex 2, France

G Thuret, S Acquart, P Gain, Cornea Bank-Blood Center of Saint Etienne, 25 Bd Pasteur, F 42055 Saint-Etienne Cedex 2, France

\section{REFERENCES}

1 EEBA. European eye bank association directory. 10th ed. 2002

2 Pels L. Organ culture: the method of choice for preservation of human donor corneas. Br J Ophthalmol 1997;81:523-5.

$3 \mathrm{Chu}$ YI, Penland RL, Wilhelmus KR. Colorimetric indicators of microbial contamination in corneal preservation medium. Cornea 2000; 19:517-20.

4 Fuller DD, Davis TE, Kibsey PC, et al. Comparison of BACTEC Plus 26 and 27 media with and without fastidious organism supplement with conventional methods for culture of sterile body fluids. J Clin Microbiol 1994;32:1488-91. 
5 Reisner BS, Woods GL. Times to detection of bacteria and yeasts in BACTEC 9240 blood culture bottles. J Clin Microbiol 1999;37:2024-6. 6 Runyon BA, Canawati HN, Akriviadis EA. Optimization of ascitic fluid culture technique. Gastroenterology 1988;95:1351-5.

7 Rayner BL, Williams DS, Oliver S. Inoculation of peritoneal dialysate fluid into blood culture bottles improves culture rates. S Afr Med J 1993;83:42-3.

8 Saubolle MA, Sewell DL, Holland MD, et al. Comparison of two commercial broth-culture systems for microbial detection in dialysates of patients on continuous ambulatory peritoneal dialysis. Diagn Microbiol Infect Dis 1989:12:457-61.

9 Von Essen R. Culture of joint specimens in bacterial arthritis. Impact of blood culture bottle utilization. Scand J Rheumatol 1997;26:293-300.

10 Yagupsky P, Dagan R, Howard CW, et al. High prevalence of Kingella kingae in joint fluid from children with septic arthritis revealed by the BACTEC blood culture system. J Clin Microbiol 1992;30:1278-81.

11 Fuller DD, Davis TE. Comparison of BACTEC plus Aerobic/F, Anaerobic/F, Peds Plus/F, and Lytic/F media with and without fastidious organism supplement to conventional methods for culture of sterile body fluids. Diagn Microbiol Infect Dis 1997;29:219-25.

12 Gain P, Thuret G, Chiquet C, et al. Use of a pair of blood culture bottles for sterility testing of corneal organ culture media. Br J Ophthalmol 2001;85:1158-62.

13 Kloess PM, Stulting RD, Waring GOd, et al. Bacterial and fungal endophthalmitis after penetrating keratoplasty. Am J Ophthalmol 1993;1 15:309-16.

14 Polack FM, Locatcher-Khorazo D, Gutierrez E. Bacteriologic study of "donor" eyes. Evaluation of antibacterial treatments prior to corneal grafting. Arch Ophthalmol 1967;78:219-25

15 Pardos GJ, Gallagher MA. Microbial contamination of donor eyes. A retrospective study. Arch Ophthalmol 1982;100:1611-3.

16 Erbezci M, Monnot PH, Michel-Briand Y, et al. Organ culture preservation of the human cornea at +31 degrees $C$ and risk of infection. J Fr Ophtalmol 1995;18:106-13.

17 Borderie VM, Laroche L. Microbiologic study of organ-cultured donor corneas. Transplantation 1998;66:120-3.
18 Leveille AS, McMullan FD, Cavanagh HD. Endophthalmitis following penetrating keratoplasty. Ophthalmology 1983;90:38-9.

19 Wiffen SJ, Weston BC, Maguire LJ, et al. The value of routine donor corneal rim cultures in penetrating keratoplasty. Arch Ophthalmol 1997:115:719-24.

20 Everts RJ, Fowler WC, Chang DH, et al. Corneoscleral rim cultures: lack of utility and implications for clinical decision-making and infection prevention in the care of patients undergoing corneal transplantation. Cornea 2001;20:586-9.

21 Ammendolia MG, Di Rosa R, Montanaro L, et al. Slime production and expression of the slime-associated antigen by staphylococcal clinical isolates. J Clin Microbiol 1999;37:3235-8.

22 Baldassarri L, Cecchini R, Bertuccini L, et al. Enterococcus spp. produces slime and survives in rat peritoneal macrophages. Med Microbiol Immunol (Berl) 2001;190:113-20.

23 Albon J, Armstrong $M$, Tullo $A B$. Bacterial contamination of human organ-cultured corneas. Cornea 2001;20:260-3.

24 Hughes JG, Vetter EA, Patel R, et al. Culture with BACTEC Peds Plus/F bottle compared with conventional methods for detection of bacteria in synovial fluid. J Clin Microbiol 2001;39:4468-71.

25 Hagenah $M$, Bohnke $M$, Engelmann K, et al. Incidence of bacterial and fungal contamination of donor corneas preserved by organ culture. Cornea 1995; 14:423-6.

26 Diamond JP, Leeming JP, Smart AD, et al. An antimicrobial effect associated with rabbit primary aqueous humour. $\mathrm{Br} J$ Ophthalmol 1994;78: 142-8

27 Bonner DP, Mechlinski W, Schaffner CP. Stability studies with amphotericin B and amphotericin B methyl ester. J Antibiot (Tokyo) 1975;28:132-5.

28 Chapman FM, Orr KE, Armitage WJ, et al. Candida glabrata endophthalmitis following penetrating keratoplasty. Br J Ophthalmol 1998;82:712-3

29 Fricker-Hidalgo $\mathbf{H}$, Chazot $F$, Lebeau B, et al. Use of simulated blood cultures to compare a specific fungal medium with a standard microorganism medium for yeast detection. Eur J Clin Microbiol Infect Dis 1998;17:113-6.

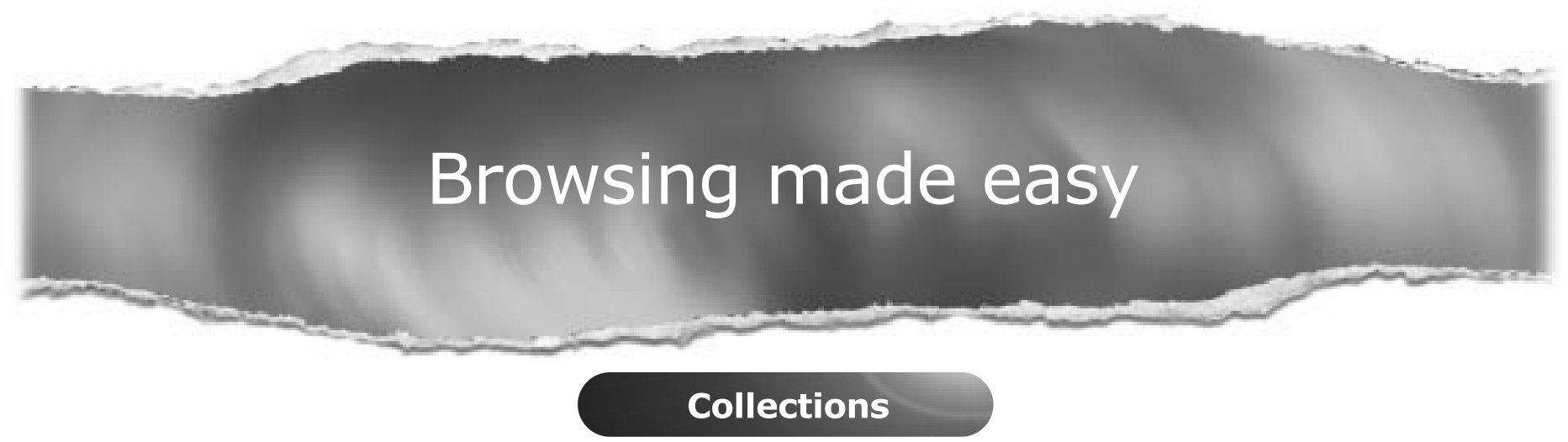

With a single click Collections allows you to find all articles that have been published in your chosen subject. Select from over 200 clinical and non-clinical topic collections and/or cross search other specialist journals, the BMJ and Cochrane Reviews

\section{www.bjophthalmol.com}

